hep-ph/9712193

Fermilab-Pub-97/425-T

December 1997

UCSD-PTH-97-37

\title{
M(ore) on Chiral Gauge Theories from D-Branes
}

\author{
Joseph Lykken ${ }^{\mathrm{a}}$, Erich Poppitz ${ }^{\mathrm{b}}$, and Sandip P. Trivedi ${ }^{\mathrm{a}}$ \\ lykken@fnal.gov, epoppitz@ucsd.edu, trivedi@fnal.gov \\ a Fermi National Accelerator Laboratory \\ P.O.Box 500 \\ Batavia, IL 60510, USA \\ ${ }^{\mathbf{b}}$ Department of Physics \\ University of California at San Diego \\ 9500 Gilman Drive \\ La Jolla, CA 92093, USA
}

\begin{abstract}
We consider a brane configuration consisting of intersecting Neveu-Schwarz five-branes, Dirichlet four-branes, and an orientifold four-plane in a $\mathbb{C}^{2} / \mathbb{Z}_{3}$ orbifold background. We show that the low-energy dynamics is described by a four dimensional gauge theory with $N=1$ supersymmetry and $S O(N+4) \times S U(N)$ or $S P(2 M) \times S U(2 M+4)$ gauge symmetry. The matter content of this theory is chiral. In particular, the $S U$ group has one matter field in the antisymmetric tensor or symmetric tensor representation and several fields in the fundamental and antifundamental representations. We discuss various consistency checks on these theories. By considering the brane configuration in M theory we deduce the spectral curves for these theories. Finally, we consider the effects of replacing the orbifold background with a non-singular ALE space (both with and without an orientifold plane) and show that it leaves the spectral curves unchanged.
\end{abstract}




\section{Introduction.}

Lately, many insights into gauge theories have emerged from the study of D-branes [1][26]. Two approaches have proved useful for this purpose. One, involving studying branes in Calabi-Yau backgrounds, was pioneered in [1], for a review see [2] and references therein. Another is to consider configurations of intersecting D-branes and NS branes [3]. As noted in [5], such configurations often correspond in M theory to a single smooth NS brane and this observation allows one to deduce the quantum behavior of the resulting gauge theory from classical considerations in $\mathrm{M}$ theory [3]-[26].

In a previous paper [21], we showed how this latter approach could be extended to study some chiral gauge theories. The key idea was to consider intersecting brane configurations in non-trivial backgrounds. Ref. [21] focused, in particular, on orbifold backgrounds. In this paper we continue the study by considering brane-configurations in a $\mathbb{Z}_{3}$ orientifold background 1 . This yields a rich class of four dimensional theories with $N=1$ supersymmetry, $S U(N) \times S O(N+4)$ or $S U(2 M+4) \times S P(2 M)$ gauge symmetry and matter in chiral representations. In particular the $S U$ group contains one field in the antisymmetric or symmetric representation and several fields in the fundamental and antifundamental representations. The analysis leading to the field theory is similar to the orbifold case in many respects but has one new feature: the orientifold carries a charge with respect to twisted RR fields which must be cancelled by D 4-branes. We show how the requirement of anomaly cancellation fixes this charge uniquely and determines the field theories.

In Section 3, we study the classical moduli space of the brane configuration and show that it agrees with the field theory analysis. In Section 4, we turn to considering the braneconfiguration in M theory and show how various non-perturbative features of the low-energy dynamics, pertaining to the spectral curves, can be derived in this way. Finally, in Section 5 , we return to the orbifold theories considered in 21] and show that the spectral curves are left unchanged (in suitable coordinates) when the orbifold background is replaced by a non-singular ALE space. This is consistent with a field theory argument showing that the curves should be independent of the Fayet-Iliopoulos terms for the anomalous $U(1)$ 's. We also make some comments with regards to orientifolds in this context.

One main motivation behind this work has been to gain a better understanding of chiral gauge theories which exhibit supersymmetry breaking. We hope some of the techniques developed here will eventually prove useful for this purpose.

\footnotetext{
${ }^{1}$ Generalising these results to $\mathbb{Z}_{N}$ backgrounds is straightforward but the resulting gauge theories are more complicated and not particularly illuminating. We will not study them here.
} 


\section{2. $\mathbb{Z}_{3}$ Orientifolds.}

\subsection{The orientifold and brane configuration.}

In this paper we will discuss a particular orientifold background with an orientifold group given by

$$
G=\left\{1, \alpha, \alpha^{2}, \Omega R(-1)^{F_{L}}, \Omega R(-1)^{F_{L}} \alpha, \Omega R(-1)^{F_{L}} \alpha^{2}\right\} .
$$

Here, $\alpha$ is a spacetime symmetry that acts on $v=X^{4}+i X^{5}$ and $w=X^{8}+i X^{9}$ as

$$
(v, w) \rightarrow\left(\alpha v, \alpha^{-1} w\right), \alpha \equiv e^{\frac{2 \pi i}{3}}
$$

$R$ is a reflection which acts on the directions transverse to the orientifold 4-plane, $X^{4}, X^{5}$, $X^{7}, X^{8}, X^{9}$, and $\Omega$ denotes world-sheet orientation reversal. The $\mathbb{Z}_{6}$ orientifold group has a $\mathbb{Z}_{2}$ subgroup:

$$
g_{1}=\left\{1, \Omega R(-1)^{F_{L}}\right\}
$$

and a $\mathbb{Z}_{3}$ subgroup

$$
g_{2}=\left\{1, \alpha, \alpha^{2}\right\} .
$$

In terms of these, the projection corresponding to the orientifold is given by

$$
\left(\frac{1+\Omega R(-1)^{F_{L}}}{2}\right)\left(\frac{1+\alpha+\alpha^{2}}{3}\right) .
$$

It is useful to bear in mind that the orientifold described above is related by T-duality to a $\mathbb{C}^{2} / \mathbb{Z}_{3}$ orbifold of Type I theory $\mathbf{L}^{2}$.

We will consider a configuration of intersecting NS and D4-branes placed in this background [5 as shown in Fig. 1. The NS branes, stretching along $X^{1}, X^{2}, X^{3}, X^{4}, X^{5}$, are placed at the orientifold point, $X^{7}=X^{8}=X^{9}=0$, and have definite positions in $X^{6}$. The D4branes stretch along $X^{1}, X^{2}, X^{3}, X^{6}$ and are also placed at $X^{7}=X^{8}=X^{9}=0$, which ensures that they intersect the NS branes. Finally, there is an orientifold 4-plane (O4-plane) parallel to the D4-branes (along $X^{1}, X^{2}, X^{3}, X^{6}$ ), which is also placed at the orientifold fixed point, $X^{4}=X^{5}=X^{7}=X^{8}=X^{9}=0$, and also intersects the NS branes.

\subsection{The gauge group and matter content: basic strategy.}

We are interested in studying the low-energy dynamics of this brane configuration. By an argument analogous to the $N=2$ case, it follows that this dynamics is governed by a

\footnotetext{
${ }^{2}$ In this paper we consider both $S O$ and $S P$ orientifolds; the above remark applies to the $S O$ case. Also we only consider non-compact backgrounds here; strictly speaking the remark on T-duality applies to a background that has been appropriately compactified.

${ }^{3}$ While, as mentioned above, the generalization to arbitrary abelian $\left(\mathbb{C}^{2} / \mathbb{Z}_{n}\right)$ orbifolds is straightforward, the nonabelian case (i.e. $\mathbb{C}^{2} / G$, with $G$-a nonabelian discrete subgroup of $S U(2)$ ) of the type considered in [28], [30], is problematic. This is because in the construction with NS branes of Fig.1 a nonabelian orbifold would mix parallel and transverse directions to the NS branes.
} 


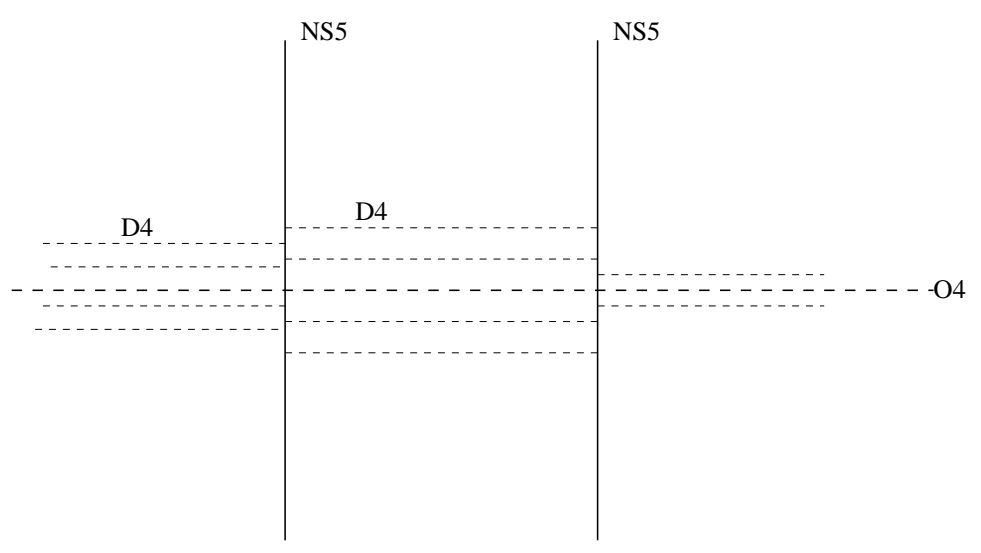

Figure 1: The brane configuration giving rise to $N=2 S O$ or $S P$ theories.

$3+1$ dimensional theory living in the intersection region of the NS and the D 4-branes, i.e., along $X^{1}, X^{2}, X^{3}$. The brane configuration in Fig. 1 leaves four supercharges unbroken, corresponding to $N=1$ supersymmetry in $3+1$ dimensions. This follows by noting that in the absence of the orientifold the configuration is invariant under eight supercharges; "turning on" the orientifold reduces this number to four.

The main question that will occupy us in this section has to do with the gauge group and matter content of this field theory. Since the discussion is somewhat involved we will first sketch out the basic strategy in this section. Following this, in section 2.3, the strategy will be explicitly implemented to find the resulting gauge group and matter content.

It is useful at the outset to summarize some conclusions from our earlier study of the field theories obtained by placing the brane configuration in Fig. 1 in orbifold backgrounds, [21]. In these cases, it was found that the resulting field theory could be obtained by starting with an $N=2$ supersymmetric theory and projecting out some states. The projection involved two operations. One was a spacetime symmetry (dictated by the particular orbifold background) and the second acted on the Chan-Paton indices. States which transformed non-trivially under this combined transformation were projected out, while those that were invariant survived and gave rise to the low-energy theory. This projection could also be understood in purely field theoretic terms in the underlying $N=2$ theory. To see this, recall that the space-time symmetries of the $N=2$ brane configuration, $S O(3)_{7,8,9} \times S O(2)_{4,5}$, appear as the classical $S U(2) \times U(1)$ R symmetry of the $N=2$ world volume theory. The orbifold spacetime symmetry then corresponded to a non-anomalous discrete subgroup of the $\mathrm{R}$ symmetriesf, while the action on the Chan-Paton factors corresponded to a discrete

\footnotetext{
${ }^{4}$ To avoid confusion, we stress that the relevant combination of $\mathrm{R}$ symmetries is non- $\mathrm{R}$ in $N=1$ terms, i.e. its action on all states in $N=1$ supermultiplets is identical.
} 
subgroup of the gauge symmetry.

For the orientifold background at hand, we also expect that the gauge group and matter content can be obtained by starting with an $N=2$ supersymmetric theory and truncating the spectrum. The $\mathbb{Z}_{2}$ subgroup of the orientifold group, eq. (2.3), suggests that the $N=2$ theory has an $S O$ or $S P$ gauge symmetry. As in the orbifold case above, the projection operator used in truncating the spectrum should have an interpretation in the field theory as the product of a discrete subgroup of the $\mathrm{R}$ symmetries and a discrete subgroup of the gauge symmetry.

The discrete subgroup of the $\mathrm{R}$ symmetry corresponds to the spacetime symmetry, eq. (2.2), and can be easily found. Identifying the appropriate subgroup of the gauge symmetry is trickier and will take more effort. This subgroup corresponds to the action on the Chan-Paton indices. The action of the group elements, $\Omega R(-1)^{F_{L}}$ and $\alpha$, eq. (2.1), on the Chan-Paton factors $\lambda$ can be represented by the matrices :

$$
\lambda \rightarrow \gamma_{\Omega R(-1)^{F_{L}}} \lambda^{T} \gamma_{\Omega R(-1)^{F_{L}}}^{-1},
$$

and

$$
\lambda \rightarrow \gamma_{\alpha} \lambda \gamma_{\alpha}^{-1}
$$

The matrices $\gamma_{\alpha}$ and $\gamma_{\Omega R(-1)^{F_{L}}}$ must furnish a representation of the orientifold group. From eq. (2.1) this implies,

$$
\begin{aligned}
\gamma_{\Omega R(-1)^{F_{L}}} & = \pm\left(\gamma_{\Omega R(-1)^{F_{L}}}\right)^{T} \\
\gamma_{\alpha}^{3} & =\text { phase. }
\end{aligned}
$$

The $+(-)$ choice in eq. (2.8) corresponds to starting with an $N=2$ theory with $S O(S P)$ symmetry. Eq. (2.9) restricts the subgroup of the gauge symmetry involved in the truncation but does not specify it uniquely. The remaining ambiguity in this choice is usually fixed by the requirement of tadpole cancellation [27 for twisted RR fields cellation condition ensures that the total charge coupling to the twisted RR field vanishes. In the present context, the RR fields come from the twisted sectors corresponding to the $\left(1, \alpha, \alpha^{2}\right)$ subgroup of the orientifold group. Since both the D4-branes and the orientifold plane can carry charge for these RR fields, the tadpole cancellation condition should provide a constraint of the form:

$$
\operatorname{Tr} \gamma_{\alpha}=- \text { orientifold charge . }
$$

\footnotetext{
${ }^{5}$ For the orientifold discussed here, the twisted RR fields involved can propagate along one spatial direction, $X^{7}$, which is of infinite extent. Even so, as argued in ref. [29], one expects the tadpoles to cancel. For our purposes this argument is suggestive; however, we actually use anomaly cancellation in the field theory to provide us with an additional constraint.
} 
The RR tadpoles are usually determined by one loop calculations, but with NS branes present no explicit conformal field theory is known and such a calculation poses a formidable challenge.

How then are we to proceed in identifying the discrete subgroup of the gauge symmetry involved in projecting out states? As was discussed in ref. 31] and more recently refs. 32, [33], one physical consequence of imposing tadpole cancellation is to ensure that the lowenergy field theory is anomaly free. Accordingly, we will, to begin with, leave the orientifold charge, eq. (2.10), to be a free parameter. Starting with an $S O$ or $S P N=2$ theory we truncate the spectrum, using the discrete subgroup of the $\mathrm{R}$ symmetry and $\gamma_{\alpha}$, and then demand that the resulting field theory is anomaly free. As we shall see, this fixes the orientifold charge uniquely and completely determines the field theory.

It is worth pointing out that, in general, anomalies on a brane world volume theory do not have to cancel. When this happens, the corresponding non-conservation of charge is due to a charge inflow from outside the brane [34], [35]. In our case, however, the gauge fields involved vanish outside the brane world volume. The non-abelian gauge anomalies in the world volume theory must therefore vanish.

As we see below, the $N=2, S O$ theory leads to a theory with $S O(N+4) \times U(N)$ gauge symmetry and $S P\left(2 M_{L}\right) \times U\left(2 M_{L}+4\right) \times S P\left(2 M_{R}\right) \times U\left(2 M_{R}+4\right)$ global symmetry. The matter content of this theory is given by:

\begin{tabular}{cc|cc|cc|cc} 
& & \multicolumn{2}{|c|}{ global } & \multicolumn{2}{c|}{ gauge } & \multicolumn{2}{|c}{ global } \\
& & $S P\left(2 M_{L}\right) \times U\left(2 M_{L}+4\right)$ & $S O(N+4) \times U(N)$ & $S P\left(2 M_{R}\right) \times U\left(2 M_{R}+4\right)$ \\
\hline $4-4$ & $\bar{Q}$ & 1 & 1 & $\square$ & $\square$ & 1 & 1 \\
strings & $A$ & 1 & 1 & 1 & $\square$ & 1 & 1 \\
\hline left & $v_{L}$ & 1 & $\square$ & $\square$ & 1 & 1 & 1 \\
strings & $q_{L}$ & 1 & $\square$ & 1 & $\square$ & 1 & 1 \\
& $\bar{q}_{L}$ & $\square$ & 1 & 1 & $\square$ & 1 & 1 \\
\hline right & $v_{R}$ & 1 & 1 & $\square$ & 1 & 1 & $\square$ \\
strings & $q_{R}$ & 1 & 1 & 1 & $\square$ & 1 & $\square$ \\
& $\bar{q}_{R}$ & 1 & 1 & 1 & $\square$ & $\square$ & 1
\end{tabular}

The $N=2, S P$ theory, on the other hand, will give rise to theories with $S P(2 M) \times$ $U(2 M+4)$ gauge symmetry and $S O\left(N_{L}+4\right) \times U\left(N_{L}\right) \times S O\left(N_{R}+4\right) \times U\left(N_{R}\right)$ global symmetry. 
The matter content is given by:

\begin{tabular}{cc|cc|cc|cc} 
& & \multicolumn{2}{|c|}{ global } & \multicolumn{2}{|c}{ gauge } & \multicolumn{2}{|c}{ global } \\
& & $S O\left(N_{L}+4\right) \times U\left(N_{L}\right)$ & $S P(2 M) \times U(2 M+4)$ & $S O\left(N_{R}+4\right) \times U\left(N_{R}\right)$ \\
\hline $4-4$ & $Q$ & 1 & 1 & $\square$ & $\overline{1}$ & 1 & 1 \\
strings & $S$ & 1 & 1 & 1 & $\square$ & 1 & 1 \\
\hline left & $v_{L}$ & $\square$ & 1 & 1 & $\overline{1}$ & 1 & 1 \\
strings & $q_{L}$ & 1 & $\square$ & 1 & $\square$ & 1 & 1 \\
& $\bar{q}_{L}$ & 1 & $\overline{1}$ & $\square$ & 1 & 1 & 1 \\
\hline right & $v_{R}$ & 1 & 1 & 1 & $\overline{1}$ & $\square$ & 1 \\
strings & $q_{R}$ & 1 & 1 & 1 & $\square$ & 1 & $\square$ \\
& $\bar{q}_{R}$ & 1 & 1 & $\square$ & 1 & 1 & $\bar{\square}$
\end{tabular}

Note that the matter content in (2.11), (2.12) is chiral. In the first case, (2.11) the $U(N)$ gauge group has an antisymmetric tensor. In (2.12) the $U(2 M+4)$ group has a symmetric tensor. (2.11) and (2.12) are among the main results of this paper.

\subsection{The explicit implementation.}

We now turn to explicitly implementing the basic strategy outlined above. It will be important to consider the $N=2$ theories with both $S O$ and $S P$ gauge groups. As was mentioned above, this corresponds to the two choices of sign in eq. (2.8). It is well known, though not well understood, from the study of $N=2$ theories that this sign flips in going across an NS brane [6], [9], [10]. In our discussion we will, correspondingly, allow for the possibility that the twisted-RR orientifold charge, eq. (2.10), also changes on crossing an NS brane. More specifically, we allow for the orientifold charges of an $S O$ and $S P$ orientifold to be different; for ease of subsequent discussion we denote these integer charges by $-C_{S O}$ and $-C_{S P}$, respectively. The corresponding tadpole cancellation conditions eq. (2.10), then are:

$$
\operatorname{Tr} \gamma_{\alpha}=C_{S O}
$$

and

$$
\operatorname{Tr} \gamma_{\alpha}=C_{S P} .
$$

We are now ready to explicitly implement the truncation procedure outlined above. We start with Fig. 1, and for concreteness first choose the orientifold charge for the 4-plane lying in between the two NS branes to correspond to an $S O$ group. After discussing this case in some detail we will then turn to an $S P$ orientifold. Let the number of D4-branes stretched between the NS branes be $N_{1}$. The orientifold charge conditions, eqs. (2.13), (2.14), mean that the configuration we start with should in general also allow for semi-infinite D4-branes stretching to the left and right of the NS branes (since the O4 plane extends both to the 
left and right of the two NS branes, semi-infinite D4 branes are needed to cancel its twistedRR charge). We take their number to be $2 N_{L}$ and $2 N_{R}$ respectively. It is useful for some purposes to think of these semi-infinite 4-branes as being limiting cases of finite ones. This can be done by adding two extra NS branes one to the far left and the other to the far right along the $X^{6}$ direction. The full gauge symmetry is then $S P\left(2 N_{L}\right) \times S O\left(N_{1}\right) \times S P\left(2 N_{R}\right)$ (in particular, this explains why the number of semi-infinite 4-branes stretching to the left and the right in Fig. 1 must be even in number). Moving the two extra NS branes to infinity turns the $S P\left(2 N_{L}\right) \times S P\left(2 N_{R}\right)$ group into a global symmetry.

Strings connecting 4-branes lying in between the two NS branes contribute a vectormultiplet of the $S O\left(N_{1}\right), N=2$ theory. In contrast, strings stretching across an NS brane with one end lying on the D4-branes stretched between the NS branes and the other end on the semi-infinite D4-branes give rise to (half) hypermultiplets which transform as $Q_{L}(\square, \square, 1)$ and $Q_{R}(1, \square, \square)$ under the $S P\left(2 N_{L}\right) \times S O\left(N_{1}\right) \times S P\left(2 N_{R}\right)$ symmetry. It is useful to describe this matter content in terms of the $N=1$ supersymmetry that eventually survives. The $N=2$ vector multiplet contains an $N=1$ vector multiplet and a chiral multiplet $\Phi_{S O}$ which transforms in the adjoint representation of the gauge group. Each half hypermultiplet gives rise to one chiral multiplet. The matter content is then given as:

\begin{tabular}{cc|c|c|c} 
& & $S O\left(N_{1}\right)$ & $S P\left(2 N_{L}\right)$ & $S P\left(2 N_{R}\right)$ \\
\hline $4-4$ & $\Phi_{S O}$ & $\boxminus$ & 1 & 1 \\
\hline left/right & $Q_{L}$ & $\square$ & $\square$ & 1 \\
strings & $Q_{R}$ & $\square$ & 1 & $\square$
\end{tabular}

The theory also has a superpotential given by:

$$
W=\operatorname{Tr} J Q_{L}^{T} \Phi_{S O} Q_{L}+\operatorname{Tr} J Q_{R}^{T} \Phi_{S O} Q_{R}
$$

where $J$ is the $S P$ invariant antisymmetric tensor $\left(J^{2}=-1\right)$. This completes our description of the $N=2$ theory.

We now turn to identifying the operators involved in projecting out states. As mentioned above, the subgroup of the $\mathrm{R}$ symmetries corresponds to a rotation by $2 \pi / 3$ in the $X^{4}, X^{5}$ and $X^{7}, X^{8}$ planes. This means that it acts on the fields as follows:

$$
\begin{aligned}
\left(A_{\mu}, \lambda\right) & \rightarrow\left(A_{\mu}, \lambda\right) \\
\Phi_{S O} & \rightarrow e^{i \frac{2 \pi}{3}} \Phi_{S O} \\
Q_{L, R} & \rightarrow e^{-i \frac{\pi}{3}} Q_{L, R}
\end{aligned}
$$

The projection operator also involves a subgroup of the gauge symmetry. More accurately, in the present context where there are semi-infinite branes present as well, it involves a subgroup of the $S P\left(2 N_{L}\right) \times S O\left(N_{1}\right) \times S P\left(2 N_{R}\right)$ symmetry. Correspondingly, there are three 
$\gamma_{\alpha}$ matrices, eq. (2.7), $\gamma_{\alpha}^{L}, \gamma_{\alpha}^{S O}, \gamma_{\alpha}^{R}$, which act on the left semininfinite D4 branes, the 4 branes stretched between the two NS branes, and the semi-infinite 4-branes stretching to the right, respectively. There is a phase ambiguity, related to these matrices, which we need to address before going further. The alert reader might have noticed that in eq. (2.9) we allowed $\gamma_{\alpha}^{3}$ to equal a phase and not be necessarily unity. The relative phases of the $\gamma_{\alpha}$ matrices corresponding to adjacent sets of D4-branes is physical: it acts nontrivially on the hypermultiplets which correspond to strings going across an NS brane and ending on these adjacent 4 branes. By a phase redefinition we can ensure that:

$$
\left(\gamma_{\alpha}^{S O}\right)^{3}=1
$$

It turns out then that

$$
\left(\gamma_{\alpha}^{L, R}\right)^{3}=-1
$$

As we saw above, the semi-infinite D4-branes can be thought of as limiting cases of finite ones with $S P$ symmetry. Keeping this in mind, our results for the respective phases can be conveniently summarised as:

$$
\left(\gamma_{\alpha}^{S O}\right)^{3}=1,\left(\gamma_{\alpha}^{S P}\right)^{3}=-1
$$

We now return to the main thread of our discussion regarding the resulting field theory. For concreteness we first consider the case where $C_{S O}, C_{S P} \geq 0$, eqs. (2.13), (2.14). This implies that (in a suitable basis) :

$$
\gamma_{\alpha}^{S O}=\operatorname{diag}\left\{1 \times \mathbf{1}_{N+C_{S O}}, \alpha \times \mathbf{1}_{N}, \alpha^{2} \times \mathbf{1}_{N}\right\}
$$

where 1 stands for an identity matrix of appropriate rank. We see that with the above choice, the number of $\mathrm{D} 4$-branes $N_{1}$ is given by:

$$
N_{1}=3 N+C_{S O}
$$

Similarly, eqs. (2.14) and (2.19) imply that:

$$
\gamma_{\alpha}^{L, R}=-\operatorname{diag}\left\{1 \times \mathbf{1}_{2 M_{L, R}}, \alpha \times \mathbf{1}_{2 M_{L, R}+C_{S P}} \alpha^{2} \times \mathbf{1}_{2 M_{L, R}+C_{S P}}\right\}
$$

Eq. (2.23) in turn means that:

$$
N_{L, R}=3 M_{L, R}+C_{S P} .
$$

\footnotetext{
${ }^{6}$ Evidence in support of this comes from adding six branes placed away from the orientifold plane and in between the two NS branes. In this case there are extra hypermultiplets, corresponding to strings stretching between the D4-branes and the 6 branes. One is lead to conclude then [27 that $\left(\gamma_{\alpha}^{(6)}\right)^{3}=-1$. Moving these 6 -branes along $X^{6}$ across an NS brane leads to the creation of D 4-branes. The $6-4$ hypermultiplets then turn into the ones discussed in the text above corresponding to strings stretched across the NS brane. This leads to the conclusion that $\left(\gamma_{\alpha}^{L, R}\right)^{3}=-1$ as well.
} 
Let us now consider the effect of the projection operators on the various fields in the theory. As we saw above the gauge boson is invariant under the $\mathbb{Z}_{3} \mathrm{R}$ symmetry. The projection operator acts on it by:

$$
A_{\mu} \rightarrow \gamma_{\alpha}^{S O} A_{\mu}\left(\gamma_{\alpha}^{S O}\right)^{-1}
$$

Eq. (2.21) shows then that the final theory has a $S O\left(N+C_{S O}\right) \times U(N)$ gauge symmetry円. Similarly the $S P\left(2 N_{L}\right)$ and $S P\left(2 N_{R}\right)$ global symmetries are broken to $U\left(2 M_{L}+C_{S P}\right) \times$ $S P\left(2 M_{L}\right)$ and $U\left(2 M_{R}+C_{S P}\right) \times S P\left(2 M_{R}\right)$ respectively. The matter content can be found in a similar manner by asking which states are invariant under the combined action of the $\mathbb{Z}_{3}$ R symmetry, $\gamma_{\alpha}^{S O}, \gamma_{\alpha}^{L}$, and $\gamma_{\alpha}^{R}$. It can be read off from eq. (2.11), after making the changes: $U\left(2 M_{L, R}+4\right) \rightarrow U\left(2 M_{L, R}+C_{S P}\right), S O(N+4) \rightarrow S O\left(N+C_{S O}\right)$.

We are now ready to impose the constraint of anomaly cancellation. Demanding that the $S U(N)$ subgroup of the $U(N)$ gauge symmetry is anomaly free gives the condition:

$$
C_{S O}-2 C_{S P}=-4
$$

So far, we have considered the case where the orientifold charge (between the NS branes) was chosen to correspond (in the $N=2$ case) to an $S O(N)$ gauge theory. Now we can consider the case where this is an $S P$ gauge symmetry instead, restricting ourselves again to the case $C_{S O}, C_{S P} \geq 0$. In this case a similar analysis reveals that the final gauge symmetry is $S P(2 M) \times U\left(2 M+C_{S P}\right)$. The global symmetry is correspondingly given by $S O\left(N_{L}+C_{S O}\right) \times U\left(N_{L}\right) \times S O\left(N_{R}+C_{S O}\right) \times U\left(N_{R}\right)$. The matter content, which survives the projection is given by eq. (2.12) after making the changes $S O\left(N_{L, R}+4\right) \rightarrow S O\left(N_{L, R}+C_{S O}\right)$ and $U(2 M+4) \rightarrow U\left(2 M+C_{S P}\right)$.

Anomaly cancellation for the $S U\left(2 M+C_{S P}\right)$ now implies that

$$
2 C_{S O}-C_{S P}=4 \text {. }
$$

Eqs. (2.26) and (2.27) now show that

$$
C_{S O}=C_{S P}=4
$$

So far we have restricted ourselves to the case $C_{S O}, C_{S P} \geq 0$. On considering the other possibilities one finds no solutions consistent with the requirements of anomaly cancellation. Thus one is lead to the unique values of the orientifold charges, eq. 2.28 )

To summarize, for an $S O$ orientifold stretched in between the two NS branes the gauge group is $S O(N+4) \times U(N)$, the global symmetry group is $S P\left(2 M_{L}\right) \times U\left(2 M_{L}+4\right) \times$

\footnotetext{
${ }^{7}$ The $U(1)$ subgroup of the $U(N)$ will turn out to be anomalous; we will return to this later on.

${ }^{8}$ While we do not do so here, one can show that the solution, eq. (2.28), is consistent with the discrete subgroup of the R symmetry, eq. (2.17), being non-anomalous as well.
} 
$S P\left(2 M_{R}\right) \times U\left(2 M_{R}+4\right)$. The matter content is as given in eq. (2.11) Note that the theory is chiral and contains and antisymmetric tensor of the $U(N)$ gauge group. The theory inherits a superpotential from eq. (2.16):

$$
W=\sum_{i=L, R} A \cdot \bar{q}_{i} \cdot \bar{q}_{i}+\bar{Q} \cdot v_{i} \cdot q_{i} .
$$

For the $S P$ orientifold the gauge symmetry is $S P(2 M) \times U(2 M+4)$, the global symmetry is $S O\left(N_{L}+4\right) \times U\left(N_{L}\right) \times S O\left(N_{R}+4\right) \times U\left(N_{R}\right)$. The matter content is shown in eq. (2.12). The corresponding superpotential is:

$$
W=\sum_{i=L, R} S \cdot v_{i} \cdot v_{i}+Q \cdot q_{i} \cdot \bar{q}_{i}
$$

We note that to prevent a global anomaly in the $S P(2 M)$ gauge symmetry $N_{L}$ and $N_{R}$ must both be either even or odd.

One final comment is in order before we end this section. As was mentioned above in the $S O$ case, the $U(1)$ subgroup of $U(N)$ is anomalous. Similarly, in the $S P$ case the $U(1)$ subgroup of $U(2 M+4)$ is anomalous. The role of anomalous $U(1)$ s in D-brane theories was discussed in ref. [28], where they were shown to be important in studying the low energy dynamics. We will not discuss this issue in great detail. The important point is that the $U(1) \mathrm{s}$ are in fact broken. The anomalies are cancelled by the Green Schwarz mechanism; the axion fields involved come from the twisted RR fields, ref. [28]. The one feature that will be important in the present discussion is that each $U(1)$ gives a $D$-term contribution to the potential energy, which is important in determining the moduli space of the theory. The axions mentioned above have partners whose expectation value corresponds to Fayet-Iliopoulos $D$ terms for the anomalous $U(1)$ s. Geometrically these expectation values correspond to blowing up the orientifold background]. We will have more to say about blowing up orbifolds and orientifolds in Section 5 .

\section{The Classical Moduli Space.}

We now turn to comparing the classical moduli space of the field theories discussed above with the allowed motions for the brane configuration. Detailed agreement between the two provides additional evidence that the correct field theories have been identified.

For simplicity we keep the number of semi-infinite 4-branes in Fig. 1 to be the minimum required by the tadpole conditions, eq. (2.13), (2.14), with eq. (2.28). This means $M_{L, R}=0$ in eq. (2.11) and the $\bar{q}_{L, R}$ fields are absent in the $S O$ case; similarly, $N_{L, R}=0$ in eq. (2.14), with both $q_{L, R}$ and $\bar{q}_{L, R}$ being absent in the SP case. The generalization for the non-minimal case is straightforward and does not add anything new.

\footnotetext{
${ }^{9}$ In fact, in the orientifold case, eqs. (2.11), (2.12), since the anomalous $U(1) \mathrm{s}$ are not traceless, a contribution to the FI terms is generated at one loop as well.
} 


\subsection{The SO theories.}

There are two cases to be discussed, corresponding to the $S O$ theories, eq. (2.11), and the $S P$ theories, eq. (2.12). We first consider the $S O$ theories. As follows from eq. (2.11), a general configuration has $3 N+4$ branes coincident with the orientifold plane. Starting with this configuration, branes can be moved away from the orientifold plane along the $X^{4}, X^{5}$ directions. Each brane, away from the orientifold plane, has five images under the $\mathbb{Z}_{6}$ orientifold group. This means that, counting images, we can move sets of six branes away from the orientifold point. Moving one set of six branes should correspond to Higgsing the gauge symmetry $S O(N+4) \times U(N) \rightarrow S O(N+2) \times U(N-2)$. Since the branes move in $X^{4}, X^{5}$ there is one complex modulus associated with this motion.

It is useful in the subsequent discussion to distinguish between two cases. If the total number of branes we started with is even, i.e. $N$ is even, this process can be carried on till $3 N$ branes have been moved away, leaving four branes stuck at the orientifold. The four branes saturate the tadpole condition, $\operatorname{Tr}\left(\gamma_{\alpha}^{S O}\right)=4$, eqs. (2.13), (2.28), and give rise to an $S O(4)$ gauge symmetry. The branes that have been moved away contribute a $U(1)^{\frac{N}{2}}$ factor, leading to a full $S O(4) \times U(1)^{\frac{N}{2}}$ gauge symmetry. There are $N / 2$ moduli parametrizing the allowed motions of the 4-branes.

If $N$ is odd, a maximum of $3(N-1)$ branes can be moved away, leaving seven branes stuck at the orientifold. These branes generically should give rise to an $S O(4)$ gauge group, leading to a full $S O(4) \times U(1)^{\frac{(N-1)}{2}}$ gauge symmetry $[$ In this case there are $(N-1) / 2$ moduli parametrising the set of allowed motions. Finally, note that in both the even and odd cases case when $N_{1}$ branes come together away from the orientifold plane (as do their images) the corresponding $U(1)^{N_{1}}$ factor in the gauge symmetry is enhanced to $U\left(N_{1}\right)$.

Let us see if the analysis in the field theory agrees with this. Note that of the field content in eq. (2.11), only the $\bar{Q}$ and $A$ fields arise from the adjoint $\Phi_{S O}$, eq. (2.15). The other fields originate from the (half) hypermultiplets $Q_{L, R}$. For the $N=2$ supersymmetric theory it is well known that the brane construction with semi-infinite 4-branes describes the Coulomb branch of the $N=2$ theory along which $\Phi_{S O}$ gets a vev but not the hypermultiplets $Q_{L, R}[9]$, [10]. Accordingly, one expects for the $N=1$ theories being discussed here that the allowed brane motion will correspond to the branch of the moduli space where only $\bar{Q}$ and $A$ get a vacuum expectation value. Below, we verify this.

For the $N$ even case the solutions to the $S O(N+4)$ and $S U(N)$ D-flatness conditions

\footnotetext{
${ }^{10} \mathrm{Eq}$. (2.11) would lead us to believe that the gauge group for the branes left at the orientifold is $S O(5) \times$ $U(1)$. However, the $U(1)$ factor is anomalous and broken; generically it also has a non-zero FI term. To meet the D-flatness condition for the $U(1)$ when the FI term is non-zero, the $\bar{Q}$ field must acquire a vev breaking $S O(5) \rightarrow S O(4)$ (see discussion after eqns. (3.3), (3.4)).
} 
are:

$$
\begin{gathered}
\bar{Q}=\left(\begin{array}{ccccccccc}
v_{1} & 0 & \ldots & 0 & 0 & 0 & 0 & 0 & 0 \\
0 & v_{1} & \ldots & 0 & 0 & 0 & 0 & 0 & 0 \\
0 & 0 & \ldots & 0 & 0 & 0 & 0 & 0 & 0 \\
\ldots & \ldots & \ldots & \ldots & \ldots & \ldots & \ldots & \ldots & \ldots \\
0 & 0 & \ldots & v_{N / 2} & 0 & 0 & 0 & 0 & 0 \\
0 & 0 & \ldots & 0 & v_{N / 2} & 0 & 0 & 0 & 0
\end{array}\right), \\
A=\operatorname{diag}\left(a_{1} \sigma_{2}, a_{2} \sigma_{2}, \ldots, a_{N / 2} \sigma_{2}\right), \text { with }\left|v_{i}\right|^{2}=2\left|a_{i}\right|^{2}+c .
\end{gathered}
$$

Since these are the only fields that get expectation values, the $F$-flatness conditions are automatically met, see eq. (2.29). The constant $c$ in eq. (3.2) is fixed, by the anomalous $U(1)$ D-flat condition, to be proportional to its Fayet-Iliopoulos term. This is easy to see upon substituting the solutions (3.1), (3.2) into the anomalous $\mathrm{U}(1)$ D-term equation, $2 \operatorname{Tr} A^{\dagger} A-$ $\operatorname{Tr} \bar{Q} \bar{Q}^{\dagger}=\xi_{F I}$. Clearly, the flat directions (3.1) and (3.2) preserve a diagonal $S O(2)^{N / 2}=$ $U(1)^{N / 2}$ abelian gauge symmetry, times a nonabelian $S O(4)$, exactly as predicted by the brane picture.

We also note that along these flat directions the superpotential, eq. (2.29), gives mass to all the $q_{L, R}$ fields and to the $S O(N)$ components of the $v_{L, R}$ fields. This can be understood in the brane picture as well: when the D4-branes moves away from the orientifold plane, the open strings connecting them to the adjacent semi-infinite 4-branes get stretched.

The $S O(4)$ group left unbroken has eight vectors and is non-asymptotically free. Consequently, in the quantum theory, we expect the $S O(4)$ gauge coupling to go to zero in the infra-red and for the massless spectrum to include the $S O(4)$ gauge bosons as well as the eight vector matter fields.

For the odd $N$ case a similar field theory analysis for the flat directions yields for the field $\bar{Q}$ :

$$
\bar{Q}=\left(\begin{array}{cccccccccc}
v_{1} & 0 & \ldots & 0 & 0 & 0 & 0 & 0 & 0 & 0 \\
0 & v_{1} & \ldots & 0 & 0 & 0 & 0 & 0 & 0 & 0 \\
0 & 0 & \ldots & 0 & 0 & 0 & 0 & 0 & 0 & 0 \\
\ldots & \ldots & \ldots & \ldots & \ldots & \ldots & \ldots & \ldots & \ldots & \ldots \\
0 & 0 & \ldots & v_{\frac{N-1}{2}} & 0 & 0 & 0 & 0 & 0 & 0 \\
0 & 0 & \ldots & 0 & v_{\frac{N-1}{2}} & 0 & 0 & 0 & 0 & 0 \\
0 & 0 & \ldots & 0 & 0 & \sqrt{c} & 0 & 0 & 0 & 0
\end{array}\right),
$$

while the antisymmetric tensor now has rank $N-1$ :

$$
A=\operatorname{diag}\left(a_{1} \sigma_{2}, a_{2} \sigma_{2}, \ldots, a_{\frac{N-1}{2}} \sigma_{2}, 0\right), \text { with }\left|v_{i}\right|^{2}=2\left|a_{i}\right|^{2}+c
$$


The constant $c$ for the odd case is also fixed to be proportional to the anomalous $U(1)$ Fayet-Iliopoulos term. Note that if the full FI term (sum of tree level and one loop) is nonzero, $c \neq 0$, and $S O(5)$ is broken down to $S O(4)$. Thus, in the odd- $N$ case generically the unbroken gauge symmetry is expected to be $U(1)^{\frac{N-1}{2}} \times S O(4)$. The superpotential gives a mass to the $q_{L, R}$ fields and the $S O(N)$ components of the $v_{L, R}$.

The Coulomb branch described above in eqns. (3.1, 3.2) (or (3.3, 3.4) for the odd- $N$ case) can also be given a gauge invariant description: from the fields $\bar{Q}$ and $A$ we can form the $U(N)$ invariant $X=A \cdot \bar{Q} \cdot \bar{Q}$, which transforms as an adjoint of $S O(N+4)$. The rank of $X$, however, is at most $N$ (or $N-1$, if $N$ is odd). Therefore, the independent $S O(N+4) \times U(N)$ invariants that describe the Coulomb branch are $\operatorname{Tr} X^{2 j}$, with $j=1, \ldots,[N / 2]$. These correspond to the positions of the $[N / 2]$ physical D4-branes away from $v=0$. Note also that the $S O(N+4) \times S U(N)$ invariant "baryon" $\bar{Q}^{2 N}$ is not allowed by the anomalous $U(1)$ (this corresponds to eliminating $c$ from (3.2) by the $\mathrm{U}(1)$ D-term). The pattern of symmetry breaking is, of course, the same as the one described above via the explicit solutions along the flat directions.

We end this section with one final comment. Above, we found that the unbroken gauge symmetry is generically, $U(1)^{\frac{N-1}{2}} \times S O(4)$. As in the even case, the $S O(4)$ has eight matter fields in the vector representation and is non-asymptotically free. Once again, in the quantum theory, we expect the $S O(4)$ gauge bosons and the eight matter fields to be present in the massless spectrum.

\subsection{The SP theories.}

The description for the $S P$ case is similar to the $S O$ case and we only present it briefly. As in the $S O$ case we expect that the allowed brane motion should correspond to the branch of moduli space where only the descendants of the $N=2$ adjoint, $Q, \bar{S}$, eq. (2.12), get an expectation value. In this case the tadpole condition, eq. (2.14) and eq. (2.28), requires $6 M+8$ branes to be coincident with the orientifold plane. $6 M$ of these can be moved away from the orientifold plane; this motion is parametrized by $M$ moduli. The remaining eight branes are stuck at the orientifold plane and saturate the tadpole condition, $\operatorname{Tr}\left(\gamma_{\alpha}^{S P}\right)=4$ (with $\gamma_{\alpha}^{S P}$ of the form given by eq. (2.23)). These eight branes give rise to an $S U(4)$ gauge symmetry, while the branes away from the orientifold plane contribute a $U(1)^{M}$ factor leading to a full $S U(4) \times U(1)^{M}$ gauge group. In field theory the moduli space is parametrised by the $M$ gauge invariants $\operatorname{Tr}(S \cdot Q \cdot Q)^{2 j}$, with $j=1, \ldots, M$. The gauge group is $S U(4) \times U(1)^{M}$ and again agrees with what was expected from brane considerations. Also, along these directions the $U(2 M)$ components of the $v_{L, R}$ fields get heavy eq. (2.30).

We saw above that a $S U(4)$ subgroup is left unbroken. It has a symmetric tensor and eight antifundamentals as matter fields and is asymptotically free. One would like to understand the quantum behavior of this theory, in particular whether it confines or not and how it global 
symmetries are realised. Unfortunately, these turn out to be difficult questions and we will have to leave them unanswered. What complicates the analysis is the fact that the theory has no flat directions. The superpotential, eq.(2.30), lifts all flat directions involving the $v_{i}$ fields leaving only one flat direction along which the field $S$ gets a vev breaking $S U(4) \rightarrow S O(4)$. This direction in turn is lifted by the $\mathrm{D}$ term of the anomalous $\mathrm{U}(1)$. We can say one important thing about the ground state of this theory: it does not break supersymmetry. One might have worried for example that along the flat direction, mentioned above, where $S$ gets a vev breaking $S U(4) \rightarrow S O(4)$, gaugino condensation in the $S O(4)$ generates a superpotential that pushes the $S$ to large vevs, in conflict with the $U(1) D$-term requirement and leading to supersymmetry breaking. However, since $S O(4) \simeq S U(2) \times S U(2)$, there is a branch along which the gaugino condensates from the two $S U(2)$ s contribute oppositely and cancel; along this branch the quantum theory has a supersymmetric vacuum.

Finally, the field theory analysis above both for the $S O$ and $S P$ cases was restricted to generic points in the moduli space. On extending it to points of enhanced gauge symmetry one finds again agreement with the expectations from the brane picture.

\section{The Hyperelliptic Curves from M Theory.}

So far in our considerations we have considered the brane configuration in the Type $I I A$ limit. We now turn to considering it in $\mathrm{M}$ theory; this will allow us to determine the spectral curves for the Coulomb phase described in section 3. Our analysis is based on the important observation of ref. [5] that in $\mathrm{M}$ theory, the brane configuration of Fig. 1 can be thought of as a single NS 5-brane which is smooth on the eleven dimensional Planck scale. In ref. [5], the $\mathrm{M}$ theory 5-brane world volume had infinite extent in the $X^{0}, X^{1}, X^{2}, X^{3}$ coordinates, while spanning a two dimensional surface in a four manifold, parametrized by $v=X^{4}+i X^{5}$ and $t=\exp \left(-\left(X^{6}+i X^{10}\right) / R\right)$. Refs. [9] and [10] extended this study to the $S O$ and $S P$ cases by introducing an orientifold 4-plane. The effects of the orientifold plane were incorporated by working in the covering space, $X^{4}, X^{5}, X^{7}, X^{8}, X^{9}$, and restricting to configurations symmetric under

$$
\left(X^{4}, X^{5}, X^{7}, X^{8}, X^{9}\right) \rightarrow-\left(X^{4}, X^{5}, X^{7}, X^{8}, X^{9}\right)
$$

For the case at hand, we consider placing the brane configuration of Fig.1 in a $\mathbb{C}^{2} / \mathbb{Z}_{3}$ orbifold background, given by identifying points as in eq. (2.2). A more convenient representation of this orbifold is obtained by embedding it as a hypersurface in $\mathbb{C}^{3}$ :

$$
y z-x^{3}=0
$$

The coordinate mapping is $y=v^{3}, z=w^{3}, x=v w$. The 5-brane is described as a curve $\Sigma$ in $C^{3} \times R^{1} \times S^{1}$. The effects of the orientifold 4-plane are incorporated, as in the $N=2$ 
case, by restricting to surfaces symmetric under $(y, w) \rightarrow-(y, w)$. The surface $\Sigma$ is smooth except at the orientifold point $y=z=x=0$ and can be parametrized by $y$ and $t$ with $z$ set equal to zero.

There is one important limitation in our discussion below that should be mentioned at the outset. We saw above that the $S O$ case gives rise to a $S O(4) \times U(1)^{\frac{[N]}{2}}$ gauge symmetry; the $S O(4)$ group is infra-red free and we expect its ultra-violet degrees of freedom to be present in the infra-red as well. The $S P$ case, on the other hand, gives rise to a $S U(4) \times U(1)^{M}$ unbroken gauge symmetry; here the inra-red behavior of the $S U(4)$ theory is difficult to analyse since it is asymptotically free and has no moduli. The discussion below will lead to the spectral curves from which the gauge couplings etc. of the $U(1)$ factors can be found, but we will not address how the physics of the $S O(4)$ or $S U(4)$ gauge theory can be deduced from $\mathrm{M}$ theory considerations. We expect this to be a fairly non-trivial question. In the Type IIA limit, the $S O(4)(S U(4))$ symmetry arose because of 4-branes stuck to the orientifold. Understanding this in $\mathrm{M}$ theory probably involves blowing up the orbifold and working in the resulting ALE space (see the discussion in section 5 in this regard), in particular the non-trivial two cycles of the ALE space should play an important role in this. We hope to return to this question in the future.

In the analysis below, our basic strategy is to start with the $N=2$ curves expressed in terms of the coordinates $t, v$. Incorporating the $\mathbb{Z}_{3}$ orbifold results in setting some of the order parameters of the $N=2$ curves to zero. Then, re-expressing the curves in terms of $t$ and $y$ gives rise to the final form of curves. The $U(1)$ gauge fields, in the resulting world volume theories, correspond to harmonic differentials odd under the $y \rightarrow-y$ symmetry and we see below that their number agrees with the field theory analysis, section 3. Some additional checks on the curves are carried out as well and shown to match field theory expectations. There are three classes of $N=2$ theories with even $S O$, odd $S O$, and $S P$ gauge symmetries, which give rise to three classes of $N=1$ theories. We consider these in turn.

\subsection{The even $S O$ case.}

To begin, we consider the $S O(2 n+4) \times U(2 n)$ gauge theory, eq. (2.11), with $M_{L}=M_{R}=0$ (incorporating additional semi-infinite four branes is trivial and does not add anything to the discussion; see eq. (4.10)). As discussed in section 2, the corresponding $N=2$ theory has $6 n+4$ branes placed between the two NS branes and eight semi-infinite branes extending to the left and right respectively. The corresponding curve is given by [9], [10]:

$$
v^{10} t^{2}+t\left(\left(v^{2}\right)^{3 n+2}+u_{2}\left(v^{2}\right)^{3 n+1}+\cdots u_{6 n+4}\right)+v^{10}=0 .
$$

Imposing the $\mathbb{Z}_{3}$ symmetry sets several terms above to zero and gives rise to the curve:

$$
v^{10} t^{2}+t\left(\left(v^{2}\right)^{3 n+2}+\tilde{u}_{1}\left(v^{2}\right)^{3 n-1}+\cdots+\tilde{u}_{n}\left(v^{2}\right)^{2}\right)+v^{10}=0 .
$$


We see that the curve above can be written as the product of two factors:

$$
v^{4}\left[v^{6} t^{2}+t\left(\left(v^{2}\right)^{3 n}+\tilde{u}_{1}\left(v^{2}\right)^{3 n-3}+\cdots+\tilde{u}_{n}\right)+v^{6}\right]=0 .
$$

The analysis in section 3.1 leads us to expect that $S O(2 n+4) \times U(2 n) \rightarrow S O(4) \times U(1)^{n}$. The discussion below shows that the second component in eq.(4.5) corresponds to the spectral curve from which the gauge couplings etc. of the $U(1)^{n}$ group can be calculated. We expect the $v^{4}$ factor to be important in understanding the $S O(4)$ unbroken symmetry, but as mentioned above we will not pursue this any further here.

Discarding the $v^{4}$ factor and substituting, $v^{3} \equiv y$ as mentioned above then gives the final form of the curve:

$$
y^{2} t^{2}+t\left(\left(y^{2}\right)^{n}+\tilde{u}_{1}\left(y^{2}\right)^{n-1}+\cdots+\tilde{u}_{n}\right)+y^{2}=0 .
$$

Eq. (4.6) meets a number of checks, as we now discuss.

First, it has $n$ order parameters, $\tilde{u}_{j}, j=1, \cdots, n$. This agrees with the field theory analysis in section 3 . The moduli $\tilde{u}_{j}$ can be related, semiclassically, to the projections of the $N=2$ moduli that are $\mathbb{Z}_{3}$ invariant: $\operatorname{Tr}\left(\Phi_{S O}^{2}\right)^{3 j}, j=1, \ldots, n$. In the $N=1$ theory, the $N=2$ adjoint decomposes into the fields $\bar{Q}$ and $A$, and using this identification, we can conclude that, semiclassically, the moduli of the $N=1$ theory are $\operatorname{Tr}\left(A \bar{Q}^{2}\right)^{2 j}$, in accord with the field theory expectations.

Second, as mentioned above, the $U(1)$ gauge bosons correspond to holomorphic differentials that are odd under $y \rightarrow-y$. One can show from eq. (4.6) that these are $n$ in number, again in accord with the field theory analysis.

Third, the curve, eq. (4.6), is consistent with all the symmetries (exact and anomalous) of the field theory. To see this it is convenient to introduce in eq. (4.6) an explicit dependence on the strong coupling scales of the $S U(2 n)$ and $S O(2 n+4)$ groups. After rescaling $t$ and $y$, dimensional analysis and the requirement of invariance with respect to the $U(1)$ (anomalous) subgroup of the $U(2 n)$ gauge symmetry fixes the curve to be:

$$
y^{2} t^{2}+t\left(\left(y^{2}\right)^{n}+\tilde{u}_{1}\left(y^{2}\right)^{n-1}+\cdots \tilde{u}_{n}\right)+\Lambda_{S U}^{2 b_{0}^{S U}} \Lambda_{S O}^{b_{0}^{S O}} y^{2}=0 .
$$

One can now verify that eq. (4.7) transforms appropriately under all the other symmetries of the field theory as well. (In eq. (4.7), $b_{0}^{S O(2 n+4)}=4 n-2$ and $b_{0}^{S U(2 n)}=4 n-5$ are the one loop beta function coefficients of the $S O(2 n+4) \times S U(2 n)$ theory, with $M_{L}=M_{R}=0$, respectively (2.11) 凹).

Finally, one can consider submanifolds of the moduli space that correspond classically to an enhanced gauge symmetry and ask if the curve reduces there to the expected form.

\footnotetext{
${ }^{11}$ We note that, since $2 b_{0}^{S U(2 n)}+b_{0}^{S O(2 n+4)}=b_{0, N=2}^{S O(6 n+4)}$, the total power of the scale dependence in eq. (4.7), $\Lambda_{S U}^{2 b_{0}^{S U}} \Lambda_{S O}^{b_{0}^{S O}}$, matches that in the $N=2$ theory, $\Lambda_{0, N=2}^{b_{0}^{S O(6 n+4)}}$.
} 
As an example, consider a situation where $n$ physical branes come close together far from the orientifold, resulting in an $U(1)^{n} \rightarrow U(n)$ enhanced symmetry. The $U(N)$ theory has an adjoint field and we expect the curve to reduce to that for an $N=2 S U(N)$ theory $\square^{\mathbb{Z}}$ along this flat direction. To see that this is indeed the case, rewrite the curve in the form:

$$
y^{2} t^{2}+t\left(y^{2}-a_{1}^{2}\right)\left(y^{2}-a_{2}^{2}\right) \ldots\left(y^{2}-a_{n}^{2}\right)+\Lambda_{S U}^{2 b_{0}^{S U}} \Lambda_{S O}^{b_{0}^{S O}} y^{2}=0
$$

The parameters $a_{i}$ in (4.8) correspond to the positions of the $n$ physical D4-branes in the $y$ plane. The configuration with enhanced $U(n)$ we described above corresponds to $a_{i}=a+\delta a_{i}$, with $\sum_{i=1}^{n} \delta a_{i}=0$; $a$ describes the center of mass motion of the $n$ physical branes in the $y$ plane, whereas the $\delta a_{i}$ are the small fluctuations around the center of mass. In the vicinity of $y=a$, i.e. for $y=a+\delta y$, the curve (4.8) becomes, keeping only the leading terms in $\delta y, \delta a_{i}$ :

$$
a^{2} t^{2}+t(2 a)^{n}\left(\delta y-\delta a_{1}\right)\left(\delta y-\delta a_{2}\right) \ldots\left(\delta y-\delta a_{n}\right)+\Lambda_{S U}^{2 b_{0}^{S U}} \Lambda_{S O}^{b_{0}^{S O}} a^{2}=0 .
$$

But this is precisely the curve describing the $S U(n)$ theory with an adjoint, with the correct strong coupling scale, as can be seen by rescaling away the coefficients in front of $t^{2}$ and $t$, and remembering that $\sum_{i=1}^{n} \delta a_{i}=0$.

We end the discussion for the $S O(2 n)$ case with two comments. First, from eq. (4.6), we see that (for $\tilde{u}_{n} \neq 0$ ) as $y \rightarrow 0, t \rightarrow 0$ and $\infty$. Thus the orientifold plane pushes the NS 5-brane out to infinity in the small $y$ region; this is analogous to what happens in the $S O(2 n) N=2$ case. Second, above we considered the case with the minimal number of semi-infinite 4 -branes, i.e. $M_{L}=M_{R}=0$. In the more general case where $M_{L}, M_{R} \neq 0$ the curve can be written down in an analogous fashion. It is given by:

$$
y^{2} t^{2} \prod_{i=1}^{M_{L}}\left(y^{2}-a_{i}^{2}\right)+e t \sum_{j=0}^{n} \tilde{u}_{i}\left(y^{2}\right)^{n-i}+f y^{2} \prod_{k=1}^{M_{R}}\left(y^{2}-b_{k}^{2}\right)=0 \text {. }
$$

\subsection{The odd SO case.}

Our discussion here will closely parallel the previous section, consequently we omit many of the details and only mention the important points. To begin, consider the theory with $S O(2 n+5) \times U(2 n+1)$ gauge symmetry and $M_{L, R}=0$, eq. (2.11). The corresponding $N=2$ theory is obtained by taking a configuration containing $6 n+74$-branes between the two NS branes and eight semi-infinite 4-branes stretching to the left and right respectively. The $N=2$ curve is :

$$
v^{9} t^{2}+t\left(\left(v^{2}\right)^{3 n+3}+u_{2}\left(v^{2}\right)^{3 n+1}+\cdots+u_{6 n+6}\right)+v^{9}=0 .
$$

${ }^{12}$ The overall $U(1)$ is not relevant for this discussion. 
Notice that this curve is invariant under the combined transformation, $v \rightarrow-v, t \rightarrow-t$. After imposing the $\mathbb{Z}_{3}$ symmetry to set various terms to zero and substituting $y \equiv v^{3}$ we get:

$$
y^{3} t^{2}+t\left(\left(y^{2}\right)^{n+1}+\tilde{u}_{1}\left(y^{2}\right)^{n}+\cdots+\tilde{u}_{n} y^{2}+\tilde{u}_{n+1}\right)+y^{3}=0 .
$$

It would seem that the curve has $n+1$ order parameters, $\tilde{u}_{j}, j=1, \cdots, n+1$; however, a semiclassical analysis shows that $\tilde{u}_{n+1}$ is not an order parameter.

Let us pause briefly to discuss this. Note that semiclassically, this parameter is the descendant of $u_{6 n+6}$ in the $N=2$ theory, eq. (4.14), which is proportional to the product of the squares of the $3 n+3$ nonzero eigenvalues of $\Phi_{S O(6 n+7)}$ (recall that the eigenvalues come in pairs). The decomposition of the adjoint of $S O(6 n+7)$ in terms of the fields $\bar{Q}, A$ has the form (in a basis where the $S O$ adjoint is an antisymmetric matrix):

$$
\Phi_{S O(6 n+7)}=\left(\begin{array}{ccc}
0 & \bar{Q}^{T} & -i \bar{Q}^{T} \\
-\bar{Q} & A & i A \\
i \bar{Q} & i A & -A
\end{array}\right)
$$

Then, substituting the solutions for the flat directions (3.3, 3.4) into (4.13), we see that $\Phi_{S O(6 n+7)}$ can at most have $3 n+1$ nonvanishing pairs of eigenvalues along the flat directions. Hence, semiclassically, $\tilde{u}_{n+1}=0$. This shows that in the full quantum theory $\tilde{u}_{n+1}$ cannot be continuously varied but leaves open the possibility that it is a constant different from zero (and dependent on the two strong coupling scales). At present, we do not know how to determine this constant from a first principles analysis. We will see below though that setting $\tilde{u}_{n+1}=0$ will yield a curve that meets several checks.

Putting $\tilde{u}_{n+1}=0$, in eq. (4.12) gives a curve which factorizes. The analysis in section 3.1 shows that generically, along the branch of moduli space under discussion here, $S O(2 n+$ $5) \times U(2 n+1) \rightarrow S O(4) \times U(1)^{n}$. In analogy with the even $S O$ curve we expect that the spectral curve for the $U(1)^{n}$ subgroup is given by discarding the overall factor of $y^{2}$. This ${ }^{\text {[3 }}$ yields the final form of the curve:

$$
y t^{2}+t\left(\left(y^{2}\right)^{n}+\tilde{u}_{1}\left(y^{2}\right)^{n-1}+\cdots+\tilde{u}_{n}\right)+y=0 .
$$

Let us describe some of the checks that the curve, eq.(4.14), meets. First, it has $n$ moduli. In fact, from eq. (4.13), it follows that semiclassically these correspond to $\operatorname{Tr}(A \bar{Q} \bar{Q})^{2 j}, j=$ $1 \cdots, n$. Second, it gives rise to $n$ holomorphic differentials odd under $y \rightarrow-y, t \rightarrow-t$. This agrees with the $n U(1)$ gauge bosons expected from the field theory analysis. Finally, after rescaling $t$ and $y$ above the curve can be written in the form:

$$
y t^{2}+t\left(\left(y^{2}\right)^{n}+\tilde{u}_{1}\left(y^{2}\right)^{n-1}+\cdots+\tilde{u}_{n}\right)+y \Lambda_{S U}^{2 b_{0}^{S U}} \Lambda_{S O}^{b_{0}^{S O}}=0,
$$

\footnotetext{
${ }^{13}$ Once again the $y^{2}$ factor should be important in understanding the $S O(4)$ symmetry in M theory.
} 
which is easily seen to be consistent with all the symmetries of the field theory.

Eq. (4.14) can also be generalized for the case $M_{L, R} \neq 0$ which correspond to having $6 M_{L}+8$ semi-infinite 4 -branes to the left and $6 M_{R}+8$ semi-infinite branes to the right. The corresponding curve is given by

$$
y t^{2} \prod_{i=1}^{M_{L}}\left(y^{2}-a_{i}^{2}\right)+e t \sum_{j=0}^{n} \tilde{u}_{j}\left(y^{2}\right)^{n-j}+f y \prod_{k=1}^{M_{R}}\left(y^{2}-b_{k}^{2}\right)=0 .
$$

\subsection{The SP case.}

Finally, we consider the case of an $S P(2 M) \times U(2 M+4)$ theory, eq. (2.12). We begin with the minimal case $N_{L, R}=0$. The corresponding $N=2$ brane configuration has $6 M+8$ 4-branes stretched between the two NS branes and four semi-infinite branes stretching to the left ad right respectively. The $N=2$ curve can now be written:

$$
v^{4} t^{2}+t v^{2}\left(\left(v^{2}\right)^{3 M+4}+u_{2}\left(v^{2}\right)^{3 M+3}+\cdots+u_{6 M+8}\right)+v^{4}=0 .
$$

Imposing the $\mathbb{Z}_{3}$ symmetry and setting appropriate terms to zero gives

$$
v^{4}\left[t^{2}+t\left(\left(v^{2}\right)^{3 M+3}+\tilde{u}_{1}\left(v^{2}\right)^{3 M}+\cdots+\tilde{u}_{M+1}\right)+1\right]=0 .
$$

Notice that the curve has factorized with two components. Generically in moduli space the $S P(2 M) \times U(2 M+4)$ symmetry is broken to $S U(4) \times U(1)^{M}$. In analogy with the $S O$ even case we expect the second component to be relevant in describing the spectral curve for the $U(1)^{M}$ group.

Discarding an overall factor of $v^{4}$ and substituting $y \equiv v^{3}$ then gives:

$$
t^{2}+t\left(\left(y^{2}\right)^{M+1}+\tilde{u}_{1}\left(y^{2}\right)^{M}+\cdots+\tilde{u}_{M}\left(y^{2}\right)+\tilde{c}\right)+1=0 .
$$

Notice that in going from eq. (4.18) to eq. (4.19) we have replaced the parameter, $\tilde{u}_{M+1}$, by a constant, $\tilde{c}$. Without this parameter the curve has $M$ moduli as required from the field theory analysis. Semiclassically, an analysis analogous to that in the $S O(2 k+1)$ case above, establishes that $\tilde{u}_{M+1}=0$. This allows for $\tilde{u}_{M+1}$ to be a constant (dependent on the two strong coupling scales) in the full quantum theory. In fact one finds that generically the curve (4.19) has $M+1$ holomorphic differentials, odd under $y \rightarrow-y$; this would imply in turn $M+1$ photons. The field theory and Type $I I A$ analysis showed that we should expect only $M$ photons. The discrepancy is resolved by setting $\tilde{c}=-2$; two branch points now coincide reducing the number of allowed photons to $M$ as required. The curve for the $S p(2 M) \times U(2 M+4)$ theory is thus finally given by:

$$
t^{2}+t\left(\left(y^{2}\right)^{M+1}+\tilde{u}_{1}\left(y^{2}\right)^{M}+\cdots+\tilde{u}_{M}\left(y^{2}\right)-2\right)+1=0 .
$$


The reader might have noticed that in above discussion, fixing the parameter $\tilde{c}$ is very similar to that involved in determining the $N=2 S P$ curves [9]. It would be nice to understand these parallels better.

The dependence of the curve (4.20) on the strong coupling scales can be made manifest. After rescaling $t$ and $y$ one finds we find:

$t^{2}+t\left(\left(y^{2}\right)^{M+1}+\tilde{u}_{1}\left(y^{2}\right)^{M}+\cdots+\tilde{u}_{M}\left(y^{2}\right)-2 \Lambda_{S P}^{b_{0}^{S P}} \Lambda_{S U}^{b_{0}^{S U}}\right)+\Lambda_{S P}^{2 b_{0}^{S P}} \Lambda_{S U}^{2 b_{0}^{S U}}=0$.

Eq. (4.21) is consistent with all the symmetries of the $S P(2 M) \times U(2 M+4)$ theory.

For the more general case where $N_{L, R} \neq 0$, eq. (2.12) the curves can be written in a straightforward manner. As mentioned before, $N_{L}$ and $N_{R}$ must both be even or odd. For the even case we get the curve:

$$
t^{2} \prod_{i=1}^{\frac{N_{L}}{2}}\left(y^{2}-a_{i}^{2}\right)+e t\left(y^{2}\left(\sum_{j=1}^{M} \tilde{u}_{j}\left(y^{2}\right)^{M-j}\right)-\tilde{c}\right)+f \prod_{k=1}^{\frac{N_{R}}{2}}\left(y^{2}-b_{k}^{2}\right)=0 .
$$

The constant $\tilde{c}$ now depends on the values of $a_{i}, b_{j}$ as well. For the odd case the curve is:

$$
t^{2} y \prod_{i=1}^{L}\left(y^{2}-a_{i}^{2}\right)+e t y^{2}\left(\sum_{j=1}^{M} \tilde{u}_{j}\left(y^{2}\right)^{M-j}\right)+f y \prod_{k=1}^{R}\left(y^{2}-b_{k}^{2}\right)=0 .
$$

We end this section with one comment about the anomalous $U(1)$ symmetries. The reader might recall that the $U(1)$ subgroup of the $U(N)$ gauge symmetry in the $S O$ case eq. (2.11), and the $U(1)$ subgroup of the $U(2 M+4)$ gauge symmetry in the $S P$ case, eq. (2.12), are anomalous. As was discussed above, this anomaly is cancelled by the shift of an axion field a. The axion supermultiplet, $\phi=\zeta+i a$, thus couples to the gauge kinetic term with a coupling $\phi W^{2}$. In effect, this coupling makes the strong coupling scales of the two groups, $\Lambda_{S U}$ and $\Lambda_{S O, S P}, \phi$ dependent. However, in the $S O$ case, we see that only a combination, $\Lambda_{S U}^{2 b_{0}^{S U}} \Lambda_{S O}^{b_{0}^{S O}}$ invariant under the anomalous $U(1)$ appears in the curve eq. (4.7), (4.15). Thus, the $\phi$ dependence drops out of the spectral curves, eq. (4.7), (4.15). A similar argument shows that the curve is $\phi$ independent in the $S P$ case as well.

The spectral curves discussed here are independent of the FI term for the anomalous $U(1)$. This follows from the above argument. The FI term gets a contribution from the vev of $\zeta$. Since the spectral curve is holomorphic, $\zeta$ can only appear in it through the combination $\phi$. The $\phi$ independence then means that the curve is completely independent of $\zeta$ and thus of the FI term.

It was argued in ref. 28 that giving a vev to $\zeta$ corresponds to blowing up the orbifold and replacing it by an ALE space. The argument above for $\zeta$ independence then also implies

\footnotetext{
${ }^{14}$ With $N_{L, R}=0$, eq. (2.12), we have $b_{0}^{S U}=4 M+5, b_{0}^{S P}=2 M+1$.
} 
that in M theory the curves should stay the same when the orbifold is replaced by an ALE space. In the next section we see that this is indeed the case.

\section{The Hyperelliptic Curves in ALE Space Backgrounds.}

In this section, we consider the effects of replacing the orbifold background with an ALE space and show that (in appropriate coordinates) it leaves the spectral curves unchanged.

We first discuss an orbifold background without an orientifold 4-plane. Towards the end we will comment on the orientifold case as well. Consider the case of a brane configuration, Fig. 1, placed in a $\mathbb{C}^{2} / \mathbb{Z}_{M}$ background, without any semi-infinite 4-branes. This configuration was studied in [21]; for completeness we summarize the main results here. The field theory corresponding to placing the brane configuration in a $\mathbb{C}^{2} / \mathbb{Z}_{M}$ background was shown to be an $S U(N)^{M}$ gauge theory with matter consisting of bifundamentals of two adjacent $S U(N)$ groups. This theory is in the Coulomb phase and the spectral curve can be written down from $\mathrm{M}$ theory considerations. To establish notation we note that the orbifold group is a spacetime symmetry which acts on $v=X^{4}+i X^{5}$ and $w=X^{8}+i X^{9}$ as:

$$
(v, w) \rightarrow\left(\alpha v, \alpha^{-1} w\right), \alpha \equiv e^{i \frac{2 \pi}{M}}
$$

The orbifold can be represented as a hypersurface in $\mathbb{C}^{3}$. After choosing coordinates, $y=v^{M}$, $z=w^{M}$, and $x=v w$, it is given by:

$$
y z=x^{M} .
$$

Note that this surface has a singularity at $y=z=x=0$.

The spectral curve for the $S U(N)^{M}$ theory (with $t=\exp \left(-\left(\frac{X^{6}+i X^{10}}{R}\right)\right)$ is now found to be:

$$
t^{2}+t\left(y^{N}+u_{1} y^{N-1}+\cdots+u_{N}\right)+1=0 .
$$

Reinstating the $\Lambda$ dependence in eq.(5.3) gives, after suitable rescalings, the curve:

$$
t^{2}+t\left(y^{N}+u_{1} y^{N-1}+\cdots+u_{N}\right)+\prod_{a=1}^{M} \Lambda_{a}^{b_{0}}=0 .
$$

One can now show, by an argument analogous to that in the previous section that this curve is independent of the FI terms for the anomalous $U(1) \mathrm{s}$. In this case, there are $M-1$ anomalous $U(1)$ s, their FI terms correspond to the $M-1$ blow-up modes of the orbifold. However, it turns out that the product of $\Lambda_{a}^{b_{0}}$ appearing in eq.(5.4) is invariant under the anomalous $U(1) \mathrm{s}$. Thus, on replacing the orbifold by a non-singular ALE space we expect that the curve, eq.(5.3) should stay the same. We turn to verifing this now. 
Repairing the orbifold singularity corresponds to replacing it by an ALE space, more specifically for the $\mathbb{Z}_{M}$ case under discussion here, a multi-center Eguchi-Hanson gravitational instanton, described by the metric [36]:

$$
\begin{aligned}
d s^{2} & =V^{-1}(d \tau+\vec{A} \cdot \overrightarrow{d x})^{2}+V d x^{2} \\
V & =\sum_{i=1}^{M} \frac{1}{\left|\vec{x}-\vec{x}_{i}\right|} \\
\vec{\nabla} V & =-\vec{\nabla} \times A,
\end{aligned}
$$

with $\tau$ being an angular variable and $x$ labeling points in $R^{3}$. By taking the limit $x \rightarrow \infty$ it is easy to see that (5.5) degenerates to the metric on $\mathbb{C}^{2} / \mathbb{Z}_{M}$ as required.

We will not use the full structure of this metric here. With a suitable choice of complex structure, the ALE space can be described as a hypersurface in $\mathbb{C}^{3}$ governed by the equation:

$$
y z=\prod_{i=1}^{M}\left(x-e_{i}\right)
$$

The coordinates $y, z$ are such that asymptotically, far from the orbifold singularity (for large $y, z), y \rightarrow v^{M}, z \rightarrow w^{M}$. Comparison with eq. (5.2) shows that in eq. (5.6) the orbifold singularity has been resolved by a complex structure deformation.

We would now like to show that the spectral curve does not change on replacing the orbifold, eq. (5.2), by the ALE space, eq. (5.6). In M theory, the spectral curve corresponds to the world volume of a single NS brane whose worldvolume has infinite extent in $X^{0}, X^{1}, X^{2}, X^{3}$ and spans a two dimensional surface in $t, y, z$, and $x$. This two dimensional surface can be described by two equations, in addition to (5.6), involving $t, y, z, x$. Asymptotically, $w \rightarrow 0$ along the 5 -brane world volume. This boundary condition can be implemented simply by identically setting

$$
z=0
$$

on the brane world volume. For consistency with eq. (5.6), we also set

$$
x=e_{a},
$$

where $e_{a}$ is one of the roots of the polynomial in eq. (5.6). This still leaves $y$ and $t$ undetermined and we need one relation between them. This equation is simply eq. (5.3) again. As in the orbifold case, it has the correct asymptotic behavior and also correctly describes various other features of the brane configuration. Thus, as promised above, we find that in going from the orbifold to the ALE space background, the M theory curve (in the coordinates $y, z$, eq. (5.6)) stays the same. 
One observation is worth making at this stage. The ALE space, eq. (5.5) has $M-1$ non-trivial two cycles. One can have an NS brane which wraps around one of these two cycles巴ّ. If such a wrapped NS brane is brought close to the curve, eq. (5.3), additional states can get massless. In our description of the curves above we did not have to confront this fact since the curve, eq.(5.3), did not contain a component consisting of such wrapped NS branes. However, these can be present in general. For example, after adding extra six branes to the brane configuration described above the theory can be in a Higgs phase (besides the Coulomb phase analysed above). The transition from the Coulomb branch to the Higgs branch involves bringing the six branes down to the orbifold singularity, one expects the extra wrapped NS branes components to be important in describing this. We leave this and related interesting issues for further study.

We end with some comments on the orientifold case. In this paper we have considered a $\mathbb{Z}_{3}$ orientifold and we restrict our remarks to this case. We saw above that the orientifold charge forces some number of 4 -branes to be stuck at the orientifold plane. A proper description of these branes in $\mathrm{M}$ theory leads us again to the questions mentioned in the last paragraph relating to extra components of the curve wrapping non-trivial two cycles. We will not be able to deal with them adequately here. However, as we saw in section 4 , the spectral curves actually factorized. One component which yielded information about the $U(1)$ gauge bosons was discussed in some detail and we argued that this component should stay the same on blowing up the orbifold. We can verify this explicitly here.

In the orientifold case, the brane configuration can be thought of as being placed in $\mathbb{Z}_{3}$ orbifold background and the effects of the orientifold 4-plane can be incorporated by ensuring that the configuration is symmetric under a reflection, eq. (4.1). We now replace the orbifold by an ALE space and would like to see how this affects the spectral curve. Most of the discussion for the orbifold case goes through here as well. The reflection symmetry, eq. (4.1), implies that the NS brane world volume is invariant under,

$$
(y, z) \rightarrow-(y, z), x \rightarrow x
$$

Eqs. (5.8), (5.7) which determine $x$ and $z$, are consistent with this requirement. Furthermore, by construction, the curves (4.6), (4.14), and (4.20) correctly incorporate the constraints coming from the orientifold 4-plane. Thus one finds that in terms of the $y, z$ variables, (5.6), the curves (4.6), (4.14), and (4.20) stay unchanged.

\section{Acknowledgments.}

We would like to thank J. Blum, M. Douglas, J. Harvey, K. Intriligator, D. Kutasov, W.

\footnotetext{
${ }^{15}$ Such a configuration can be conveniently seen if the orbifold singularity is resolved by a Kähler deformation. For the ALE space, eq. (5.5), the Kähler deformations are equivalent to complex structure deformations.
} 
Skiba and E. Witten for discussions. The research of JL and ST is supported by the Fermi National Accelerator Laboratory, which is operated by the Universities Research Association, Inc., under contract no. DE-AC02-76CHO3000. E.P. was supported by DOE contract no. DOE-FG03-97ER40506.

\section{References}

[1] A. Klemm, W. Lerche, P. Mayr, C. Vafa, and N. Warner, "Self-Dual Strings and $N=2$ Supersymmetric Field Theory", hep-th/9604034, Nucl. Phys. B477 (1996) 477.

[2] A. Klemm, "On the Geometry Behind $N=2$ Supersymmetric Effective Actions in Four Dimensions", hep-th/9705131.

[3] A. Hanany and E. Witten, "Type IIB Superstrings, BPS Monopoles, and ThreeDimensional Gauge Dynamics", hep-th/9611230, Nucl. Phys. B492 (1997) 152.

[4] S. Elitzur, A. Giveon and D. Kutasov, "Branes and $N=1$ Duality in String Theory," hep-th/9702014, Phys. Lett. B400 (1997) 269.

[5] E. Witten, "Solutions of Four-Dimensional Field Theories via $M$ Theory", hepth/9703166, Nucl. Phys. B500 (1997) 3.

[6] N. Evans, C.V. Johnson, and A. D. Shapere, "Orientifolds, Branes, and Duality of 4D Gauge Theories", hep-th/9703210, Nucl. Phys. B505 (1997) 251.

[7] J.H. Brodie and A. Hanany, "Type IIA Superstrings, Chiral Symmetry, and $N=14 \mathrm{D}$ Gauge Theory Dynamics", hep-th/9704043.

[8] S. Elitzur, A. Giveon, D. Kutasov, E. Rabinovici, and A. Schwimmer, "Brane Dynamics and $N=1$ Supersymmetric Gauge Theory," hep-th/9704104, Nucl. Phys. B505 (1997) 202.

[9] K. Landsteiner, E. Lopez, and D. Lowe, " $N=2$ Supersymmetric Gauge Theories, Branes and Orientifolds", hep-th/9705199.

[10] A. Brandhuber, J. Sonnenschein, S. Theisen, and S. Yankielowicz, " $M$ Theory and Seiberg-Witten Curves: Orthogonal and Simplectic Groups", hep-th/9705232, Nucl. Phys. B504 (1997) 175.

[11] K. Hori, H. Ooguri, and Y. Oz, "Strong Coupling Dynamics of Four-Dimensional $N=1$ Gauge Theories from $M$ Theory Fivebrane," hep-th/9706082. 
[12] E. Witten, "Branes and the Dynamics of QCD", hep-th/9706109.

[13] A. Brandhuber, N. Itzhaki, V. Kaplunovsky, J. Sonnenschein, and S. Yankielowicz, "Comments on the $M$ Theory Approach to $N=1$ SQCD and Brane Dynamics," hepth/9706127, Phys. Lett. B410 (1997) 27.

[14] A. Hanany, M.J. Strassler, and A. Zaffaroni, "Confinement and Strings in MQCD", hep-th/9707244.

[15] T. Nakatsu, K. Ohta, T. Yokono, and Y. Yoshida, "Higgs Branch of $N=2$ SQCD and $M$ Theory Branes", hep-th/9707258.

[16] M. Schmaltz and R. Sundrum, " $N=1$ Field Theory Duality from $M$-theory," hepth/9708015.

[17] J. de Boer and Y. Oz, "Monopole Condensation and Confining Phase of $N=1$ Gauge Theories Via $M$ Theory Fivebrane," hep-th/9708044.

[18] C. Csaki and W. Skiba, "Duality in $S p$ and $S O$ Gauge Groups from $M$ Theory," hepth/9708082.

[19] K. Landsteiner and E. Lopez, "New Curves from Branes", hep-th/9708118.

[20] C. Ahn, K. Oh, and R. Tatar, " $S p\left(N_{c}\right)$ Gauge Theories and $M$ Theory Fivebrane," hep-th/9708127; " $M$ Theory Fivebrane Interpretation for Strong Coupling Dynamics of $S O\left(N_{c}\right)$ Gauge Theories," hep-th/9709096; "M Theory Fivebrane and Confining Phase of $N=1 S O\left(N_{c}\right)$ Gauge Theories, hep-th/9712005.

[21] J. Lykken, E. Poppitz, and S.P. Trivedi, "Chiral Gauge Theories from D-Branes", hepth/9708134, Phys. Lett. B, in press.

[22] A. Giveon and O. Pelc, " $M$ Theory, Type IIA String and $4 \mathrm{D} N=1 \mathrm{SUSY} S U\left(N_{L}\right) \times$ $S U\left(N_{R}\right)$ Gauge Theory," hep-th/9708168.

[23] H. Ooguri, "M Theory Fivebrane and SQCD," hep-th/9709211.

[24] P.S. Howe, N.D. Lambert, and P.C. West, "Classical M-Fivebrane Dynamics and Quantum $N=2$ Yang-Mills", hep-th/9710034.

[25] J. de Boer, K. Hori, H. Ooguri, and Y. Oz, "Kähler Potential and Higher-Derivative Terms From $M$ Theory Fivebrane", hep-th/9711143.

[26] N.D. Lambert and P.C. West, "Gauge Fields and $M$-Fivebrane Dynamics", hepth/9712040. 
[27] E. Gimon and J. Polchinski, "Consistency Conditions for Orientifolds and D-Manifolds", hep-th/9601038, Phys. Rev. D54 (1996) 1667.

[28] M. Douglas and G. Moore, "D-Branes, Quivers, and ALE Instantons", hep-th/9603167.

[29] J. Blum and K. Intriligator, "Consistency Conditions for Branes at Orbifold Singularities", hep-th/9705030, Nucl. Phys. B506 (1997) 223.

[30] J. Blum and K. Intriligator, "New Phases of String Theory and 6d RG Fixed Points via Branes at Orbifold Singularities", hep-th/9705044, Nucl. Phys. B506 (1997) 199.

[31] J. Polchinski and Y. Cai, "Consistency of Open Superstring Theories", Nucl. Phys. B296 (1988) 91.

[32] E. Gimon and C. Johnson, "K3 Orientifolds", hep-th/9604129, Nucl. Phys. B477 (1996) 715.

[33] A. Dabholkar and J. Park, "Strings on Orientifolds", hep-th/9604178, Nucl. Phys. B477 (1996) 701.

[34] C. Callan and J. Harvey, "Anomalies and Fermion Zero Modes on Strings and Domain Walls", Nucl. Phys. B250 (1985) 427.

[35] J. Blum and J. Harvey, "Anomaly Inflow for Gauge Defects", hep-th/9310035, Nucl. Phys. B416 (1994) 119.

[36] T. Eguchi, P.B. Gilkey, and A.J. Hanson, "Gravitation, Gauge Theories and Differential Geometry", Phys. Rep. 66 (1980) 213. 